ÉGYPTE monde arabe

\section{Égypte/Monde arabe}

$21 \mid 1995$

Économie égyptienne et perspectives de paix au

Proche-Orient

\title{
Conséquences financières du programme de stabilisation et d'ajustement structurel sur les secteurs productifs (1991-1993)
}

\section{Ramzi Zaki}

\section{(2) OpenEdition}

\section{Journals}

Édition électronique

URL : https://journals.openedition.org/ema/404

DOI : 10.4000/ema.404

ISSN : 2090-7273

Éditeur

CEDEJ - Centre d'études et de documentation économiques juridiques et sociales

Édition imprimée

Date de publication : 31 mars 1995

Pagination : 166-173

ISSN : 1110-5097

Référence électronique

Ramzi Zaki, «Conséquences financières du programme de stabilisation et d'ajustement structurel sur les secteurs productifs (1991-1993) », Égypte/Monde arabe [En ligne], 21 | 1995, mis en ligne le 08 juillet 2008, consulté le 07 juillet 2022. URL : http://journals.openedition.org/ema/404 ; DOI : https://doi.org/ 10.4000/ema.404

Ce document a été généré automatiquement le 7 juillet 2022.

Tous droits réservés 


\section{Conséquences financières $\mathrm{du}$ programme de stabilisation et d'ajustement structurel sur les secteurs productifs (1991-1993)}

Ramzi Zaki

\section{NOTE DE L'ÉDITEUR}

Traduit de l'arabe par Samia Rizq.

1 La signature par le gouvernement égyptien, en mai et novembre 1991, de deux accords de stabilisation économique et d'ajustement structurel avec le Fonds monétaire international et la Banque mondiale, est le résultat de la politique d'ouverture entamée en 1974 et des déséquilibres économiques qu'elle a engendrés : augmentation du déficit de la balance des paiements et de la dette extérieure, dont le service absorbait à lafin des années 80 l'essentiel des recettes d'exportation, déficit croissant du budget de l'État et hausse du taux d'inflation, dépréciation de la livre égyptienne et dollarisation, baisse des taux d'épargne, d'investissement et de croissance, élévation du taux du chômage, détérioration du niveau de vie d'une fraction importante des Égyptiens, aggravation des inégalités de revenu et de patrimoine. Cette signature, puis la stricte observance des deux programmes par le gouvernement égyptien sous contrôle périodique du FMI et la Banque mondiale, conditionnaient l'annulation de $50 \%$ des dettes officielles de l'Égypte (soit environ 10 milliards de dollars), le rééchelonnement du solde au Club de Paris et l'obtention de nouveaux crédits à des conditions favorables.

2 Les recommandations des programmes de stabilisation et d'ajustement structurel, élaborés et suivis de façon coordonnée par le FMI et la Banque Mondiale sur le principe de la cross conditionality ${ }^{1}$, se fondent sur l'analyse selon laquelle les problèmes économiques des pays en développement résultent de l'application passée de politiques 
économiques ne prenant pas en compte les variables externes. Le programme de stabilisation vise ainsi à réduire à court terme le déficit extérieur et la part du déficit budgétaire dans lePIB ainsi qu'à ralentir l'inflation par la contraction de la masse monétaire et la dévaluation. Quant au programme d'ajustement structurel, il propose de rétablir une croissance forte en supprimant les distorsions de prix et l'intervention de l'État, et d'accroître la capacité de l'économie à s'adapter aux chocs extérieurs par une plus grande ouverture. De fait, ces programmes visent davantage à accroître la capacité de remboursement des pays du tiers-monde qu'à favoriser leur développement ou à résoudre leurs problèmes économiques et sociaux. Par nature récessifs, ces programmes induisent en effet une modification radicale de la structure économique et sociale parle biais de la libération des prix et de l'importation, par la promotion des exportations et la suppression de la propriété publique, le plus souvent au prix d'importants sacrifices sociaux

\section{L'effet de la stabilisation sur les secteurs productifs égyptiens}

3 Après trois ans d'application, le programme de stabilisation a abouti en Égypte, comme dans d'autres pays en développement, à la haussé des taux d'intérêt créditeurs et débiteurs, au plafonnement du crédit, à la dévaluation de la livre égyptienne et à la libéralisation des changes. Il a également conduit à l'augmentation des recettes de l'État grâce à la hausse des impôts et des taxes, ainsi qu'au relèvement des prix des produits et services du secteur public (en particulier celui de l'énergie). Il a enfin permis dé ralentir le taux de croissance des dépenses publiques par la suppression des subventions; la réduction de l'emploi, et de l'investissement dans le secteur public,et ce, afin de limiter, le déficit budgétaire.

4 La hausse des taux d'intérêt induite par l'émission des bons du Trésor a fait diminuer l'investissement sans accroître l'épargne, qui stagne autour de 8\% du PNB depuis 1991. Le renchérissement du crédit a seulement favorisé un déplacement de l'épargne d'une forme à l'autre sans en accroître le volume global. L'émission massive de bons du Trésor à un taux de rémunération élevé exonéré d'impôts a fait de l'État le principal emprunteur sur le marché monétaire, attirant tant les banques, les caisses de retraite et les entreprises excédentaires que les ménages aisés. Plutôt que de s'orienter vers l'investissement et d'accroître les capacités de production, l'épargne a servi à financer le déficit courant de l'État et la consommation publique. Selon la Banque centrale ${ }^{2}$, la part de la FBCF (formation brute de capital fixe) dans le PNB a chuté de 19,8\% en $1991 / 92^{3}$ à $18,6 \%$ en 1992/93, malgré une croissance de 1,7\% au prix du marché en 1992/93. La croissance du PNB, inférieure à celle de la population, est due essentiellement aux services qui augmentent deux fois plus rapidement que les biens industriels et agricoles (voir tableau 1 en annexe).

5 Faute de disposer de recul et de données suffisantes pour apprécier l'impact de la stabilisation et de l'ajustement sur le secteur productif, nous faisons l'hypothèse, sur la base des rares données existantes, que les deux secteurs, public et privé, ont également subi la forte hausse des coûts de production et la stagnation économique. De fait, les politiques monétaires et financières de l'ajustement structurel ont enlevé toute efficacité aux avantages fiscaux accordés depuis le début de l'ouverture économique aux investissements étrangers et locaux. 

entreprises publiques, jamais évoqué par les technocrates des organisations internationales et les partisans locaux de la privatisation, est de voir le capital étranger dominer à nouveau le destin du pays en contrôlant ses plus grandes entreprises. Le pouvoir politique risque de se trouver sous la domination du capital étranger, qui aura des intérêts vitaux à défendre en Égypte, comme ce fut le cas avant la révolution du 23 juillet 1952.

8 Par ailleurs, la cession progressive aux capitaux étrangers des actifs publics entraînera une augmentation parallèle de la part du revenu national susceptible d'être transférée à l'étranger, réduisant d'autant le revenu net et le niveau de vie des Égyptiens, aggravant le déficit de la balance des paiements et menaçant la parité de la livre égyptienne, les fragiles réserves accumulées depuis 1991 et le niveau des prix intérieurs. De plus, les multinationales qui, seules, peuvent assumer le risque de participer aux privatisations, pourraient dissimuler leurs bénéfices ou faire jouer la corruption pour réduire leurs impôts locaux. Enfin, les actifs publics risquent d'être revendus à des pays tiers hostiles à la sécurité nationale et aux intérêts stratégiques de l'Égypte. Les privatisations risquent également d'accentuer le chômage. Les déclarations du gouvernement adressées à la population, garantissant l'emploi et les droits des travailleurs, contredisent les promesses faites aux acheteurs potentiels, qui affirment leur totale liberté en matière de main-d'œuvre et de salaires. Moyen de redistribution des ressources nationales au profit du secteur privé local et international, la privatisation affaiblit l'Etat en le privant de l'excédent économique qui lui permettait de réaliser ses objectifs économiques, sociaux et sa sécurité.

\section{Conclusion}

9 Les politiques monétaires et financières en vigueur en Égypte depuis la signature de l'accord avec le FMI et la Banque mondiale en 1991 ont permis de stabiliser le taux de change de la livre, de freiner l'inflation et de réduire le déficit budgétaire. En contrepartie, et conformément à leur objectif de stabilisation de la demande globale, elles ont ralenti l'activité et l'investissement. De surcroît, les mesures de libéralisation $\mathrm{du}$ commerce extérieur ont augmenté l'exposition des secteurs productifs à la concurrence extérieure, tandis que le processus de privatisation qui s'engage comporte des risques importants pour l'autonomie nationale. La politique économique en cours, dont les bons résultats monétaires et financiers sont indéniables, devra être modifiée à l'avenir pour permettre d'accroître le taux de croissance, de réduire le chômage et d'augmenter le niveau de vie des Égyptiens.

10 Notes des p. 168-169.

(4) Créé pour assurer la restructuration et la privatisation de l'ancien secteur public, transformé en «secteur des affaires " par la loi 203 de 1991. Voir Business monthly (NDLR).

(5) Ainsi le gouvernement français a-t-il vendu en avril 1993. par le biais de la Coface (qui détenait 27 milliards $\mathrm{FF}$ soit 5 milliards $\mathrm{S}$ de créances vis-à-vis de l'Égypte), 100 millions $\$$ de créances à des banques françaises à $50 \%$ de leur valeur nominale, que la Banque centrale égyptienne s'engage à racheter. La Bank of America et d'autres ont fait une offre d'achat des dettes égyptiennes mises en vente par des organismes publics,

Égypte/Monde arabe, 21 | 1995 
britanniques et belges en vue de garantir leurs créances. La presse a indiqué que le succès d'une telle opération conduirai! de nombreux gouvernements à convertir la totalité de leurs créances sur l'Égypte en participations dans des projets publics.

(6) En Égypte, la gestion des grands hôtels est déjà assurée par des firmes hôtelières privées étrangères. Leur privatisation ne modifierait donc pas cette gestion.

\section{ANNEXES}

Tableau 1 : Taux de croissance du PIB et taux de FBCF*

\begin{tabular}{|l|l|l|l|l|}
\hline Secteur / Taux de croissance réel (prix 1991/92) & $\begin{array}{l}1991 / 92 \\
\text { définitif }\end{array}$ & $\begin{array}{l}1992 / 93 \\
\text { préliminaire }\end{array}$ & $\begin{array}{l}1992 / 93 \\
\text { définitif }\end{array}$ & $\begin{array}{l}1993 / 94 \\
\text { préliminaire }\end{array}$ \\
\hline Secteur des biens & 1,7 & 1.6 & 2,3 & 3,8 \\
\hline Agriculture & 2.0 & 2,9 & 2.5 & 3,4 \\
\hline \hline Industrie et métallurgie & 1,5 & 2,7 & 2,9 & 4,1 \\
\hline Pétrole & 1.8 & 4,1 & 1,6 & 3,7 \\
\hline \hline Electricité & 4,6 & 4,6 & 3,4 & 4,1 \\
\hline Construction & 0,7 & $(-11,9)$ & 1,0 & 4.6 \\
\hline Secteur des services & 1.9 & 2.9 & 2,0 & 2,9 \\
\hline \hline Transports et communications & 1,0 & 6,2 & 6.2 & 6.6 \\
\hline Canal de Suez & 2,0 & 4,2 & 4,0 & 3,8 \\
\hline \hline Commerce & 1,8 & 3,6 & 2.9 & 4,0 \\
\hline \hline Finances & 5.3 & 2,5 & 3,9 & 5,1 \\
\hline \hline Assurances & 5,3 & $(-11,4)$ & 4,3 & $(-18,6)$ \\
\hline \hline Tourisme, restauration, hôtellerie & 3.4 & 4.2 & 4.0 & 4,4 \\
\hline \hline Immobilier & & 5.3 & 4,3 & 6,8 \\
\hline
\end{tabular}




\begin{tabular}{|l|l|l|l|l|}
\hline Assurances sociales & 2,1 & 5,4 & 5.7 & 4,3 \\
\hline Services gouvernementaux & 4,2 & 4,3 & 4.2 & 4,0 \\
\hline Services privés & 1,2 & 3,7 & 3.5 & 4,1 \\
\hline P!B & 1,9 & 2,4 & 2.5 & 3.6 \\
\hline Taux de FBCF & 19,8 & 18,6 & 20.2 & 20,8 \\
\hline
\end{tabular}

*FBCF : formation brute de capital fixe. Source : Banque centrale, Rapport annuel 1992/93, pp. 10-14 et 1993/94, pp. 8-12.

Tableau 2 : Structure des exportations par produits (millions S)

\begin{tabular}{|c|c|c|c|c|c|c|c|c|}
\hline & \multicolumn{2}{|c|}{$\begin{array}{l}\text { 1991/92 } \\
\text { définitif }^{1}\end{array}$} & \multicolumn{2}{|c|}{$\begin{array}{l}\text { 1992/93 } \\
\text { préliminaire }\end{array}$} & \multicolumn{2}{|c|}{$\begin{array}{l}\text { 1992/93 } \\
\text { définitif }\end{array}$} & \multicolumn{2}{|c|}{$\begin{array}{l}\text { 1993/94 } \\
\text { préliminaire }\end{array}$} \\
\hline & Valeur & $\%$ & Valeur & $\%$ & Valeur & $\%$ & Valeur & $\%$ \\
\hline Total & $3.633,5$ & 100,0 & $3.417,3$ & 100,0 & $3.416,7$ & 100,0 & $3.064,8$ & 100,0 \\
\hline Produits agricoles & 257,5 & $7,1-$ & 198,2 & 5,8 & 199,4 & 5.8 & 238,2 & 7,8 \\
\hline Coton & 35,4 & 1,0 & 36,8 & 1,1 & 36.7 & 1.1 & 45,0 & 1,5 \\
\hline Autres produits agricoles & 222.1 & 6,1 & 161,4 & 4,7 & 162,7 & 4,7 & 193,2 & 6,3 \\
\hline Industrie pétrolière & $1.651,1$ & 45,4 & $1.802,9$ & 52,8 & $1.802,9$ & 52,8 & $1.499,4$ & 48.9 \\
\hline Pétrole brut & 1.172 .0 & $32^{\wedge}$ & 1279,4 & 37,5 & 1279.4 & 37,5 & 932.1 & 30,4 \\
\hline Produits pétroliers & 479,1 & 13,2 & 523,5 & 15,3 & 523,5 & 15,3 & 567,3 & 18,5 \\
\hline Produits industriels & $1.461,1$ & 40,2 & 1.141 .6 & "33,4 & 1.167 .4 & 342 & $1.127,4$ & 36,8 \\
\hline Textiles & 575,4 & 15,8 & 449,7 & 13,2 & 450,9 & 132 & 495,5 & 16,2 \\
\hline Autres produits industriels & 885,7 & 24,4 & 691.9 & 20,2 & 716,5 & 21,0 & 631,9 & 20,6 \\
\hline Produits non classés & 263,8 & 7,3 & 274.6 & 8,0 & 247.0 & 7,2 & 199,8 & 6,5 \\
\hline
\end{tabular}

Source : Banque centrale, Rapport annuel 1992/93, p. 94 et 1993/94, p. 66

\section{NOTES}

1. «Cross conditionality » : chacune des deux organisations exige la signature concomitante d'un accord avec l'autre avant de consentir un prêt. 
2. Rapport annuel de la Banque centrale d'Égypte, 1992/93, p. 13.

3. Année financière du 1er Juillet au 30 Juin.

\section{AUTEUR}

\section{RAMZI ZAKI}

Institut national de la planification 